\title{
Targeting Moderate and Severe Male Stress Urinary Incontinence With Adjustable Male Slings and the Perineal Artificial Urinary Sphincter: Focus on Perioperative Complications and Device
} Explantations

\author{
Alexander Kretschmer ${ }^{1, \star}$, Tanja Hüsch ${ }^{2, \star}$, Frauke Thomsen ${ }^{3}$, Dominik Kronlachner ${ }^{3}$, Alice Obaje ${ }^{4}$, Ralf Anding ${ }^{5}$, \\ Fabian Queissert ${ }^{13}$, Carsten M. Naumann ${ }^{14}$, Carola Wotzka ${ }^{15}$, Torben Hofmann ${ }^{16}$, Roland Seiler ${ }^{17}$, Axel Haferkamp ${ }^{2}$, \\ Ricarda M. Bauer ${ }^{1}$; Debates On Male Incontinence (DOMINO)-Project \\ ${ }^{1}$ Department of Urology, Ludwig-Maximilians-University, Campus Großhadern, Munich, Germany \\ ${ }^{2}$ Department of Urology, University Medical Center of Johannes-Gutenberg University, Mainz, Germany \\ ${ }^{3}$ Department of Urology and Pediatric Urology, University Hospital Frankfurt, Frankfurt, Germany \\ ${ }^{4}$ Department of Urology, St. Bernward Hospital Hildesheim, Hildesheim, Germany \\ ${ }^{5}$ Department of Urology and Pediatric Urology, University Hospital Bonn, Bonn, Germany \\ ${ }^{6}$ Department of Urology, Asklepios Hospital West Hamburg, Hamburg, Germany \\ ${ }^{7}$ Department of Urology and Pediatric Urology, Helios Hospital Duisburg, Duisburg, Germany \\ ${ }^{8}$ Department of Urology, Hospital Lueneburg, Lueneburg, Germany \\ ${ }^{9}$ Department of Urology, Hospital Göttlicher Heiland Vienna, Vienna, Austria \\ ${ }^{10}$ Department of Urology, Hospital Weinviertel Korneuburg, Korneuburg, Austria \\ ${ }^{11}$ Department of Urology and Pediatric Urology, St. Barbara Hospital Hamm GmbH, Hamm, Germany \\ ${ }^{12}$ Department of Urology, Evangelic Hospital Bielefeld, Bielefeld, Germany \\ ${ }^{13}$ Department of Urology, University Hospital Muenster, Muenster, Deutschland \\ ${ }^{14}$ Department of Urology and Pediatric Urology, University Hospital Schleswig-Holstein, Campus Kiel, Kiel, Germany \\ ${ }^{15}$ Department of Urology, Diakonie Hospital Stuttgart, Stuttgart, Germany \\ ${ }^{16}$ Department of Urology, Diakonie Hospital Schwäbisch Hall, Schwäbisch Hall, Germany \\ ${ }^{17}$ Department of Urology, University Hospital Bern, Bern, Switzerland
} Tobias Pottek ${ }^{6}$, Achim Rose ${ }^{7}$, Roberto Olianas ${ }^{8}$, Alexander Friedl ${ }^{9}$, Wilhelm Hübner ${ }^{10}$, Roland Homberg ${ }^{11}$, Jesco Pfitzenmaier ${ }^{12}$,

Corresponding author: Alexander Kretschmer (iD https://orcid.org/0000-0002-6511-4354 Department of Urology, Ludwig-Maximilians-University, Campus Großhadern, Marchioninistrasse 15, 81377 Munich, Germany

E-mail: Alexander.kretschmer@med.uni-muenchen.de / Tel: +49-89-4400-0 / Fax: +49-89-4400-5444

*Alexander Kretschmer and Tanja Hüsch contributed equally to this study as cofirst authors.

Submitted: May 6, 2016 / Accepted after revision: June 30, 2016 
Purpose: To analyze perioperative complications and postoperative explantation rates for selected readjustable male sling systems and the perineal single-cuff artificial urinary sphincter (AUS) in a large, contemporary, multi-institutional patient cohort. Methods: Two hundred eighty-two male patients who underwent implantation between 2010 and 2012 in 13 participating institutions were included in the study ( $\mathrm{n}=127$ adjustable male sling [ $\mathrm{n}=95$ Argus classic, $\mathrm{n}=32$ Argus T], $\mathrm{n}=155$ AUS). Perioperative characteristics and postoperative complications were analyzed. The explantation rates of the respective devices were assessed using the Fisher exact test and the Mann-Whitney U-test. A Kaplan-Meier curve was generated. Potential features associated with device explantation were analyzed using a multiple logistic regression model $(\mathrm{P}<0.05)$.

Results: We found significantly increased intraoperative complication rates after adjustable male sling implantation (15.9\% [adjustable male sling] vs. $4.2 \%$ [AUS], $\mathrm{P}=0.003)$. The most frequent intraoperative complication was bladder perforation $(\mathrm{n}=17)$. Postoperative infection rates did not vary significantly between the respective devices $(\mathrm{P}=0.378)$. Device explantation rates were significantly higher after AUS implantation (9.7\% [adjustable male sling] vs. $21.5 \%$ [AUS], $\mathrm{P}=0.030)$. In multivariate analysis, postoperative infection was a strong independent predictor of decreased device survival (odds ratio, 6.556; $\mathrm{P}=0.001$ ).

Conclusions: Complication profiles vary between adjustable male slings and AUS. Explantation rates are lower after adjustable male sling implantation. Any kind of postoperative infections are independent predictors of decreased device survival. There is no significant effect of the experience of the implanting institution on device survival.

Keywords: Urinary Incontinence, Stress; Urinary Sphincter, Artificial; Complications

- Research Ethics: The study was performed according to the Helsinki Declaration and approved by the Ethics Committee of the Medical Faculty of Johann-Wolfgang Goethe University, Frankfurt am Main, Germany (\#442/13). Written informed consent was obtained from all subjects.

- Conflict of Interest: R. M. Bauer declares consultancy work, lectures, and participation in clinical trials for AMS (Minnetonka, MN, USA) and Promedon (Cordoba, Argentina). T. Pottek declares consultancy work, lectures for AMS, Zephyr and Teleflex. C.M. Naumann declares consultancy work, lectures, and participation in clinical trials for Coloplast. The remaining authors have nothing to disclose.

\section{INTRODUCTION}

The reported prevalence of stress urinary incontinence (SUI) in men is high and increases with age [1]. When conservative treatments like pelvic floor muscle training fail, a surgical approach is recommended [2]. Due to its high success rates in the treatment of male SUI, the artificial urinary sphincter (AUS) is still the standard treatment for persistent moderate to severe SUI [2,3]. Nowadays, the AMS 800 device (Boston Scientific, formerly AMS, Minnetonka, MN, USA) is most commonly used, even though there are alternative devices available [4]. However, revision rates for nonmechanical reasons such as erosion, urethral atrophy, and infections range from $7 \%$ to $17 \%$ [58]. In addition, manual dexterity and sufficient mental function is necessary to handle the AUS properly. Several readjustable male slings have been introduced in recent years and patient demand for slings is high [9]. The Argus classic system (Promedon, Cordoba, Argentina) is a radiopaque cushioned system with a silicone foam pad for soft compression of the bulbar urethra and is implanted via a retropubical approach [10]. The Argus T sling system (Promedon) was launched in 2008 and differs in terms of implantation route (transobturatoric approach) and the position of the washers [11].
Since we are still lacking prospective comparative trials investigating different surgical devices, the "Debates on Male Incontinence" (DOMINO) working group aims to provide large comparative multicenter studies providing robust data on daily routine at various Central European continence centers. In addition, evidence is currently based on case series from a few high-volume centers. However, in Central Europe, occasional implanters implant a significant proportion of devices. In the current study, we compare perioperative complications as well as short-term explantation rates of some of the most commonly used adjustable male sling systems with the current gold standard, the perineal single-cuff AUS in a large retrospective, international, multicenter comparative study.

\section{MATERIALS AND METHODS}

\section{Patient Population}

Inclusion criteria in the current study were as follows: verified nonneurogenic SUI, moderate or severe SUI (defined as daily pad usage of $\geq 3$ ), and implantation of an Argus classic male sling, Argus T male sling, or perineal single-cuff AMS 800 AUS. In total, 282 patients from 13 different institutions were included. 


\section{Study Design and Data Assessment}

The study was approved by a local ethics committee. Data collection was performed retrospectively by external independent physicians who were not members of the implanting center.

Perioperative complications were defined as any complications occurring within the first 6 months postoperatively. Analyzed perioperative complications included bleeding, wound healing disorders, acute urinary retention, infection, and prolonged perineal pain (lasting more than 6 weeks postoperatively). Infection was defined as any recorded infection due to clinical presentation (fever, local tenderness, erythema, skin fixation, abscess, pathologic urinary test results) not including primary device infections. Acute urinary retention was defined as the complete inability to void. In addition, device explantation rates were assessed. Furthermore, in order to define potential features associated with device explantation, multiple hypothesized patient- and procedure-derived risk factors were tested.

\section{Patient Selection}

Due to the retrospective study design, patient selection within the respective groups was not standardized. Instead, the following basic operating principles were used. All included patients were considered not to be suitable for a retrourethral transobturator male sling based on the medical history, volume of urine leakage, existence of nocturnal incontinence, or a failed urethrocystoscopic repositioning test [12]. Patients who were not physically or mentally able to handle the AUS were automatically offered an adjustable male sling. If patients were considered to be able to handle the AUS properly, they were offered the option of either an adjustable male sling or an AUS. The final decision was then mostly based on the patient's decision as well as on the expertise of the treating physician.

\section{Statistical Analysis}

The primary endpoint was the perioperative complication rate as well as device explantation rate after adjustable male sling and AUS implantation. For categorical data, the Fisher exact test and chi-square test were used. For continuous data, the Wilcoxon rank sum test was performed. Univariate analysis was performed to test for potential predictive features for device explantation. Additionally, a multivariate analysis using a multiple logistic regression model was performed. To further display potential differences in explantation rates, a Kaplan-Meier curve was generated. All statistical analyses as well as graphics were created using IBM SPSS Statistics ver. 23.0 (IBM Co., Ar- monk, NY, USA). A P-value $<0.05$ was considered to be statistically significant.

\section{RESULTS}

\section{Pre- and Perioperative Patient Characteristics and Perioperative Standard Procedures}

Table 1 summarizes the baseline characteristics of 282 consecutive patients ( $\mathrm{n}=127$ adjustable male sling [ $\mathrm{n}=95$ Argus classic, $\mathrm{n}=32$ Argus T], $\mathrm{n}=155$ AUS) that were included in the study. Briefly, patients undergoing adjustable male sling implantation were less likely to undergo salvage surgery $(21.3 \%$ vs. $38.1 \%$, $\mathrm{P}=0.003$ ) and less likely to have a history of bladder neck stricture (5.5\% vs. $12.9 \%, \mathrm{P}=0.042)$ compared to patients who underwent AUS implantation.

Every patient received intraoperative antibiotic prophylaxis. Antibiotic prophylaxis was started one day preoperatively in $27.3 \%$ of the cases $(7.9 \%$ [adjustable male sling] vs. $43.2 \%$ [AUS], $\mathrm{P}<0.001)$. An intraoperative single-shot antibiotic prophylaxis was administered in $8.2 \%$ of all patients $(14.2 \%$ [adjustable male sling] vs. $3.2 \%$ [AUS], $\mathrm{P}<0.001$ ).

\section{Perioperative Complications and Explantation Rates}

To improve the ability to generalize our results, perioperative complication rates of the respective devices were analyzed in a selected subgroup of our patient cohort. The median number of device implantations per institution in our study cohort was found to be 20.5 (range, 1-52). Consequently, the participating centers were divided into less-experienced centers ( $\leq 20 \mathrm{im}$ plantations between 2010 and 2012; defined as "low-volume") and more experienced centers ( $>20$ implantations; defined as "high-volume"). Implantations that had been performed by a less-experienced center $(n=49)$ were excluded from this particular analysis.

Perioperative complications and explantation rates after adjustable male sling and AUS implantation of the remaining patient cohort are summarized in Table 2. We found significantly increased intraoperative complication rates after adjustable male sling implantation (15.9\% [adjustable male sling] vs. $4.2 \%$ [AUS], $\mathrm{P}=0.004)$. The most frequent intraoperative complication was bladder perforation $(n=17)$. Postoperative infection rates did not vary significantly between the respective devices $(\mathrm{P}=0.378)$. The most frequently observed infections were urinary tract infections $(n=6)$, followed by wound infections $(\mathrm{n}=3)$, and fever without an apparent focus $(\mathrm{n}=2)$. 
Follow-up data concerning explantation rates were available for 207 of 233 patients (88.8\%). Device explantation rates were significantly higher after AUS implantation (9.7\% [adjustable male sling] vs. $21.5 \%$ [AUS], $\mathrm{P}=0.030$ ). A Kaplan-Meier sur-

Table 1. Patient characteristics of 282 patients that were included in the current study.

\begin{tabular}{|c|c|c|c|c|}
\hline Characteristic & Adjustable male sling & AUS & Total & $\mathrm{P}$-value \\
\hline No. of patients & $127(45.0)$ & $155(55.0)$ & $282(100)$ & - \\
\hline Age (yr) & $70.0 \pm 6.9$ & $69.4 \pm 10.9$ & $69.7 \pm 9.4$ & 0.601 \\
\hline Body mass index $\left(\mathrm{kg} / \mathrm{m}^{2}\right)$ & $27.4 \pm 3.3$ & $27.7 \pm 4.1$ & $27.5 \pm 3.6$ & 0.730 \\
\hline Salvage surgery & $27(21.3)$ & $59(38.1)$ & $86(30.5)$ & $0.003^{*}$ \\
\hline Pelvic radiation & $35(27.6)$ & $36(23.2)$ & $71(25.2)$ & 0.412 \\
\hline Urethral stricture & $21(16.5)$ & $39(25.2)$ & $60(21.3)$ & 0.082 \\
\hline Bladder neck stricture & $7(5.5)$ & $20(12.9)$ & $27(9.6)$ & $0.042^{*}$ \\
\hline Diabetes mellitus & $29(22.8)$ & $28(18.1)$ & $57(20.2)$ & 0.372 \\
\hline $\begin{array}{l}\text { Etiology of SUI } \\
\text { PPI } \\
\text { TURP } \\
\text { Other }\end{array}$ & $\begin{array}{c}110(86.6) \\
14(11.0) \\
3(2.4)\end{array}$ & $\begin{array}{c}127(81.9) \\
24(15.5) \\
4(2.6)\end{array}$ & $\begin{array}{c}237(84.3) \\
38(13.5) \\
7(2.5)\end{array}$ & $\begin{array}{l}0.250 \\
0.316 \\
1.000\end{array}$ \\
\hline $\begin{array}{l}\text { Grade of SUI } \\
\text { Moderate } \\
\text { Severe }\end{array}$ & $\begin{array}{l}82 / 115(71.3) \\
33 / 115(28.7)\end{array}$ & $\begin{array}{l}62 / 96(64.6) \\
34 / 96(35.4)\end{array}$ & $\begin{array}{r}144 / 211(68.2) \\
67 / 211(31.8)\end{array}$ & $\begin{array}{l}0.303 \\
0.303\end{array}$ \\
\hline Pads per $24 \mathrm{hr}$ & $5.9 \pm 2.6$ & $6.9 \pm 3.3$ & $6.3 \pm 3.5$ & 0.198 \\
\hline
\end{tabular}

Values are presented as number (\%) or mean \pm standard deviation.

AUS, artificial urinary sphincter; SUI, stress urinary incontinence; PPI, postprostatectomy incontinence; TURP, transurethral resection of the prostate.

${ }^{\star} \mathrm{P}<0.05$, statistically significant.

Table 2. Perioperative complication rates after 233 device implantations in high-volume (>20 implantations in the study cohort) centers

\begin{tabular}{lcccc}
\hline Variable & Adjustable male slings & AUS & Total & P-value \\
\hline No. of patients & $113(48.5)$ & $120(51.5)$ & $233(100)$ & - \\
Intraoperative complications & $18(15.9)$ & $5(4.2)$ & $23(9.9)$ & $7(3.0)$ \\
Postoperative bleeding & $2(1.8)$ & $5(4.2)$ & $6(2.6)$ & $0.004^{*}$ \\
Postoperative wound healing disorder & $2(1.8)$ & $4(3.3)$ & $24(10.3)$ & 0.276 \\
Postoperative urinary retention & $11(9.7)$ & $13(10.8)$ & $26(11.2)$ & 0.442 \\
Prolonged perineal pain & $17(15.0)$ & $9(7.5)$ & $12(5.2)$ & 0.670 \\
Postoperative infection & $4(3.5)$ & $20(21.5)$ & $31 / 207(15.0)$ & 0.095 \\
Device explantation & $11(9.7)$ & $4(3.3)$ & & 0.378 \\
Reasons for device explantation & & $6(5.0)$ & $7(3.0)$ & $0.030^{*}$ \\
Persistent SUI & $3(2.7)$ & $6(5.0)$ & $7(3.0)$ & 0.533 \\
Device infection & $1(0.9)$ & $3(2.5)$ & $3(1.3)$ & 0.070 \\
Urethral erosion & $1(0.9)$ & $0(0)$ & $1(0.4)$ & 0.070 \\
Mechanical device failure & $0(0)$ & $1(0.8)$ & $3(1.3)$ & 0.135 \\
Persistent urinary retention & $1(0.9)$ & $179.2 \pm 165.4$ & $189.3 \pm 165.9$ & 0.612 \\
Other & $2(1.8)$ & $216.8 \pm 172.8$ & & 0.533 \\
Time to explantation (day) & &
\end{tabular}

Values are presented as number (\%) or mean \pm standard deviation.

AUS, artificial urinary sphincter; SUI, stress urinary incontinence.

a) Total $\mathrm{n}=207$ due to lost-to-follow-up patients. ${ }^{\star} \mathrm{P}<0.05$, statistically significant. 
vival curve was generated (Fig. 1). We performed a separate subgroup analysis within the adjustable male sling group and compared perioperative complication rates after Argus classic and after Argus $\mathrm{T}$ implantation. We found that all intraoperative complications occurred during Argus classic implantation $(\mathrm{P}=0.006)$. Perineal pain occurred significantly more frequently after Argus T implantation (4.7 [Argus classic] vs. 46.4\% [Argus $\mathrm{T}], \mathrm{P}<0.001)$.

\section{Risk Factors for Device Explantation}

We next evaluated various predefined patient- and procedurederived features that were hypothesized to contribute to increased device explantation rates. To address the potential impact of the experience of the implanting center, high-volume as

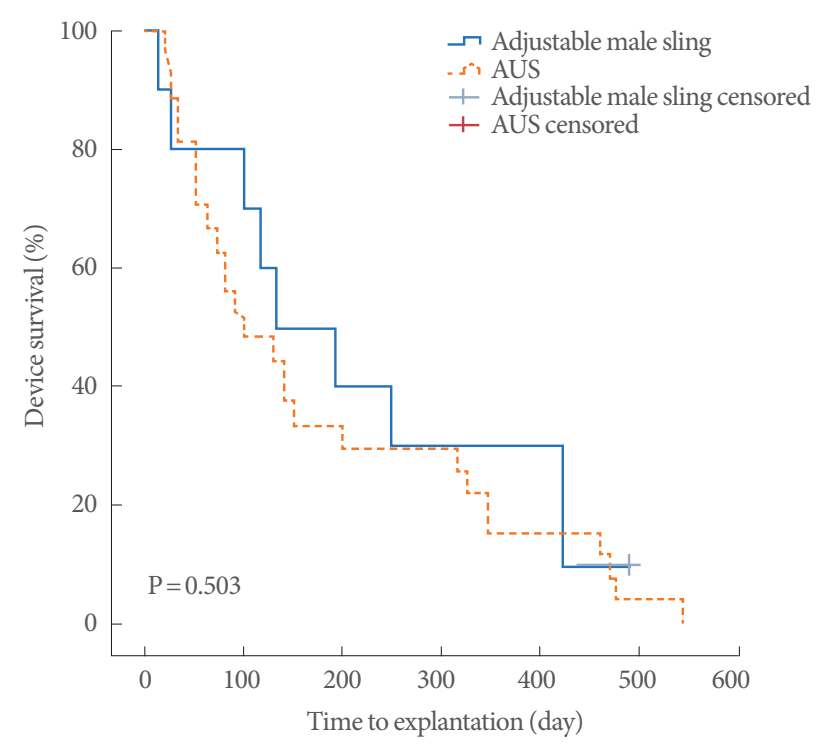

Fig. 1. Kaplan-Meier plot comparing device survival after adjustable male sling (blue line: median survival 130 days; 95\% confidence interval [CI], 7-255) to AUS (red dots: median survival 101 days, $95 \%$ CI, 27-183) implantation. AUS, artificial urinary sphincter. well as low-volume centers were included in the analysis.

Results of the univariate analysis are summarized in Table 3. No significant impact of preoperative age, BMI, or preoperative grade of SUI based on daily pad use was observed $(\mathrm{P}=0.474$, $\mathrm{P}=0.090, \mathrm{P}=0.462$, respectively).

Based on the results of the univariate analysis, a multivariate analysis was performed using a multiple logistic regression model. Hereby, all features showing significant results in the univariate analysis, the respective devices and the experience of the implanting centers were included.

The results of the multivariate analysis are summarized in Table 4. To summarize, any kind of postoperative infection was a strong independent predictor of decreased device survival (odds ratio [OR], 6.559; $\mathrm{P}=0.001$ ), and there was a trend to-

Table 3. Univariate analysis of predefined hypothesized predictive features for increased or decreased device explantation rates

\begin{tabular}{lcc}
\hline Predictive feature & $\begin{array}{c}\text { Device explantation } \\
\text { rate (\%) }\end{array}$ & P-value \\
\hline High-volume centre & 12.0 & 0.457 \\
Diabetes mellitus & 22.0 & 0.824 \\
Postprostatectomy incontinence & 11.8 & 0.323 \\
Pelvic radiation & 18.3 & 0.148 \\
Salvage surgery & 14.0 & 0.701 \\
Bladder neck stricture & 25.9 & $0.040^{\star}$ \\
Urethral stricture & 10.0 & 0.663 \\
Intraoperative single-shot antibiotics & 17.4 & 0.512 \\
Intraoperative complications & 16.7 & 0.525 \\
Postoperative bleeding & 18.2 & 0.638 \\
Postoperative wound healing disorder & 33.3 & 0.094 \\
Postoperative urinary retention & 17.9 & 0.377 \\
Prolonged perineal pain & 21.9 & 0.154 \\
Postoperative infection & 46.7 & $0.001^{\star}$ \\
\hline
\end{tabular}

${ }^{\star} \mathrm{P}<0.05$, statistically significant.

Table 4. Summary of the multivariate analysis using a binary logistic regression model, indicating any kind of postoperative infections being independent prognostic features for increased device explantation rates

\begin{tabular}{lcccc}
\hline Predictive feature & SE & P-value & Odds ratio & 95\% CI \\
\hline Artificial urinary sphincter & 0.424 & 0.074 & 2.615 & $1.138-6.009$ \\
Bladder neck stricture & 0.544 & 0.361 & 6.644 & $0.566-4.779$ \\
Postoperative infection & 0.592 & 0.001 & 0.559 & $2.055-20.934$ \\
High-volume centre & 0.455 & 0.615 & 0.795 & $0.326-1.941$ \\
\hline
\end{tabular}

SE, standard error; CI, confidence interval. 
wards increased explantation rates in the AUS subgroup (OR, $2.615 ; \mathrm{P}=0.074$, ).

\section{DISCUSSION}

Based on current therapy algorithms, the perineal single-cuff AUS is the gold standard in the treatment of moderate and severe male SUI $[2,4]$. Adjustable male slings are currently of emerging interest in this patient group, but evidence regarding their use is still weak. In the current multi-institutional study, we compare perioperative safety as well as explantation rates of 2 adjustable sling systems (Argus classic and Argus T) and the perineal single-cuff AMS 800 in a large, multi-institutional contemporary patient cohort.

To address differences in postoperative complication and device explantation rates in an adequately generalizable way, we focused on patients that have undergone surgery in a sufficiently experienced incontinence center.

We found a significantly increased intraoperative complication rate during adjustable male sling implantation compared to AUS implantation. When performing a subgroup analysis of the adjustable male sling patient cohort, we found that the vast majority of intraoperative complications happened to be bladder perforations during Argus classic implantation. Addressing this topic, Bochove-Overgaauw and Schrier [13] analyzed the outcome after 100 Argus classic implantations and described bladder perforations in $6 \%$. Considering the different surgical approaches, it seems intuitive that bladder perforations occur much less frequently during Argus $\mathrm{T}$ implantation [11].

A statistical trend towards prolonged perineal pain in the adjustable male sling group could be observed. Further subgroup analysis revealed significantly increased postoperative perineal pain rates in the Argus T cohort. This is in line with previous prospective case series [11]. The explanation for higher postoperative perineal pain rates after transobturatoric male sling implantation is supposed to be multifactorial, including neuropathic pain. Since treatment of persistent perineal pain is often challenging, our findings highlight the importance of preoperative patient information [11].

In univariate analysis, device explantation rates were significantly higher after AUS implantation compared to adjustable male sling implantation. Time-to-explantation did not vary significantly. While considerable explantation rates after AUS implantation have been reported previously, our results highlight the safety of the analyzed adjustable male slings [5]. However,
AUS implantation could not be confirmed as an independent risk feature for device explantation in the current study and confounding factors such as experience of the implanting center cannot be completely ruled out.

In line with previous results, urethral erosion was the most common reason for AUS explantation in our patient cohort [5]. Persistent SUI and device dislocation were the most frequent reasons for adjustable male sling explantation. The peripheric washers of the Argus T male sling dislocated in 2 cases, leading to ineffectiveness and inability to readjust properly. In 1 case, the perineal cushion of an Argus T sling dislocated laterally, and it eventually had to be explanted.

A main finding of the current study is the analysis of features associated with device explantation rates. We found a more than 6 fold increased explantation risk after any kind of perioperative infection, such as UTI, epididymitis, or wound infections. Our results highlight the importance of avoidance of perioperative infections by the implanting team.

Our results indicate that a history of bladder neck stricture might have a moderate impact on device survival. Literature regarding treatment of male SUI and concomitant bladder neck stricture is currently limited to small case series [14]. Anger et al. [15] reported general feasibility of simultaneous stricture incision and AUS implantation without stating the detailed functional outcomes after the implantations. Mark et al. [16] reported a social continence rate (no pads or one thin pad per day) of 92\% for patients with concomitant anastomotic stricture treated with AUS implantation.

Evidence addressing risk factors for explantation after adjustable male sling implantation is still rare. Dalpiaz et al. [17] studied potential risk factors for general complication rates after Argus classic implantation and found no statistically significant impact of median age, comorbidity, incontinence grade, or previous pelvic irradiation. In contrast, Bochove-Overgaauw and Schrier [13] observed increased complication rates after Argus classic implantation in previously irradiated patients and patients with a history of urethral stricture disease.

In the current study, we provide evidence that the experience of the respective center might not be a major prognostic feature of device survival. Our cutoff value may be debatable. However, it is based on the contemporary caseload of 13 international continence centers. These findings are of the utmost clinical importance since in many parts of the world, including in Central Europe, occasional implanters perform a majority of the incontinence device implantations. 
Our study is not devoid of limitations. First and foremost are the limitations inherent to retrospective analyses. However, it remains unclear if the heterogeneity of the analyzed subgroups may have affected the outcome of our study to some degree. Due to its retrospective, multi-institutional design, patient selection and postoperative follow-up was not standardized in the current study. It is also unclear how the individual surgeon's learning curve and experience may have affected the complication rates in our multicenter patient collective [18]. We focused on short-term complications within 6 months. It is therefore possible that long-term complication profiles may vary from the ones observed in this study. The Argus T subgroup was smaller than the Argus classic and AUS subgroup. The results of the current study must be confirmed in larger patient cohorts.

In summary, we observed different perioperative complication profiles for adjustable male slings and the perineal AUS. Postoperative infections are an independent predictive feature for decreased device survival. The experience of the implanting center did not correlate significantly with device explantation rates. This is the first comparative study that includes several devices in a large contemporary, multi-institutional patient cohort. We analyzed low-volume institutions separately and are therefore able to reflect the current standard of care for when occasional implanters perform the implantations.

\section{REFERENCES}

1. Lucas MG, Bosch RJ, Burkhard FC, Cruz F, Madden TB, Nambiar $\mathrm{AK}$, et al. EAU guidelines on assessment and nonsurgical management of urinary incontinence. Eur Urol 2012;62:1130-42.

2. Lucas MG, Bosch RJ, Burkhard FC, Cruz F, Madden TB, Nambiar $\mathrm{AK}$, et al. EAU guidelines on surgical treatment of urinary incontinence. Eur Urol 2012;62:1118-29.

3. Herschorn $S$. The artificial urinary sphincter is the treatment of choice for post-radical prostatectomy incontinence. Can Urol Assoc J 2008;2:536-9.

4. Kretschmer A, Hübner W, Sandhu JS, Bauer RM. Evaluation and management of postprostatectomy incontinence: a systematic review of current literature. Eur Urol Focus 2016;2:245-59.

5. Van der Aa F, Drake MJ, Kasyan GR, Petrolekas A, Cornu JN; Young Academic Urologists Functional Urology Group. The artificial urinary sphincter after a quarter of a century: a critical systematic review of its use in male non-neurogenic incontinence. Eur Urol 2013;63:681-9.
6. Raj GV, Peterson AC, Toh KL, Webster GD. Outcomes following revisions and secondary implantation of the artificial urinary sphincter. J Urol 2005;173:1242-5.

7. Elliott DS, Barrett DM. Mayo Clinic long-term analysis of the functional durability of the AMS 800 artificial urinary sphincter: a review of 323 cases. J Urol 1998;159:1206-8.

8. Lai HH, Hsu EI, Teh BS, Butler EB, Boone TB. 13 years of experience with artificial urinary sphincter implantation at Baylor College of Medicine. J Urol 2007;177:1021-5.

9. Kumar A, Litt ER, Ballert KN, Nitti VW. Artificial urinary sphincter versus male sling for post-prostatectomy incontinence--what do patients choose? J Urol 2009;181:1231-5.

10. Romano SV, Metrebian SE, Vaz F, Muller V, D’Ancona CA, Costa DE Souza EA, et al. An adjustable male sling for treating urinary incontinence after prostatectomy: a phase III multicentre trial. BJU Int 2006;97:533-9.

11. Bauer RM, Rutkowski M, Kretschmer A, Casuscelli J, Stief CG, Huebner W. Efficacy and complications of the adjustable sling system Argus T for male incontinence: results of a prospective 2-center study. Urology 2015;85:316-20.

12. Bauer RM, Gozzi C, Roosen A, Khoder W, Trottmann M, Waidelich R, et al. Impact of the 'repositioning test' on postoperative outcome of retroluminar transobturator male sling implantation. Urol Int 2013;90:334-8.

13. Bochove-Overgaauw DM, Schrier BP. An adjustable sling for the treatment of all degrees of male stress urinary incontinence: retrospective evaluation of efficacy and complications after a minimal followup of 14 months. J Urol 2011;185:1363-8.

14. Cox A, Herschorn S. Management of the incontinent patient with a sphincteric stricture following radical prostatectomy. Curr Opin Urol 2014;24:578-85.

15. Anger JT, Raj GV, Delvecchio FC, Webster GD. Anastomotic contracture and incontinence after radical prostatectomy: a graded approach to management. J Urol 2005;173:1143-6.

16. Mark S, Pérez LM, Webster GD. Synchronous management of anastomotic contracture and stress urinary incontinence following radical prostatectomy. J Urol 1994;151:1202-4.

17. Dalpiaz O, Knopf HJ, Orth S, Griese K, Aboulsorour S, Truss M. Mid-term complications after placement of the male adjustable suburethral sling: a single center experience. J Urol 2011;186:604-9.

18. Sandhu JS, Maschino AC, Vickers AJ. The surgical learning curve for artificial urinary sphincter procedures compared to typical surgeon experience. Eur Urol 2011;60:1285-90. 